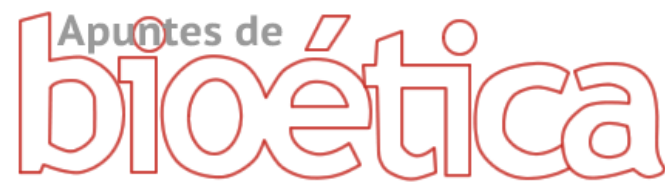

https://doi.org/10.35383/apuntes.v2i2.289

\title{
La bioética como herramienta para enfrentar el bullying en la Educación Básica Regular
}

\author{
José Rogelio Ruiz Alvarado ${ }^{1}$
}

\section{INFORMACIÓN DEL ARTÍCULO RESUMEN}

Historia del artículo:

Recibido el 25 de setiembre de 2019

Aceptado el 20 de diciembre de 2019

\section{Palabras claves: \\ Transversalidad \\ Bioética \\ Educación Básica Regular \\ Disfuncionalidad familia \\ Bullying}

En cualquier situación que un país enfrente y represente una problemática social, la educación será siempre una de las mejores armas de lucha para acabar con ella. La Educación Básica Regular en el Perú, ha cambiado en las últimas décadas, ahora ya no se restringe solamente a la adquisición de contenidos curriculares, sino que ahora cuenta con los llamados ejes transversales, que se convierten en un trasfondo del proceso educativo incluyendo así temas con relevancia social y orientados a la difusión de los valores y principios buscando siempre el bien común.

Los estudiantes de EBR enfrentan, además de sus propias responsabilidades académicas y los cambios propios de su etapa, una gran problemática: el acoso escolar o más conocido Bullying, el cual a través de sus distintas manifestaciones atentan directamente contra la dignidad humana de cada estudiante.

La interiorización que realizarán los estudiantes de los principios bioéticos gracias a la transversalidad en los planes educativos, permitirá que ellos mismos identifiquen qué situación representa alguna vulneración o lesión de su dignidad humana y tomar las medidas para superarlas con la ayuda de las personas preparadas para ello.

${ }^{1}$ Licenciado en Ciencias Históricas Sociales y Filosofía, Segunda Especialidad en Psicopedagogía, Mtro. En Docencia y Gestión Educativa, estudios en Bioética y Biojurídica, Docente en Educación Básica Regular de la Institución Educativa Santa Lucía - Ferreñafe, Docente de Pregrado y Coordinador Académico de Maestría en Bioética y Biojurídica de la Universidad Católica Santo Toribio de Mogrovejo USAT. Email: joserogelio_ruiz@hotmail.com ORCID: https://orcid.org/0000-0003-16832787 


\section{Bioethics As A Tool To Face Bullying In The EBR.}

\section{ABSTRACT}

\section{Keywords:}

Transversality

Bioethics

Regular Basic Education

Family dysfunctionality

Bullying

\begin{abstract}
In any situation that a country faces and represents a social problem, education will always be one of the best weapons of struggle to end it. Regular Basic Education in Peru has changed in recent decades, now it is not only restricted to the acquisition of curricular content, but now it has the so-called transversal axes, which become a background of the educational process including issues With social relevance and oriented to the dissemination of values and principles, always seeking a common good.

EBR students face, in addition to their own academic responsibilities and the changes of their stage, a great problem: bullying or better known Bullying, which through its different manifestations directly threaten their human dignity.

The internalization that students will carry out of the bioethical principles thanks to the transversality in the educational plans, allows them to identify which situation represents any violation or injury of their human dignity and take the measures to overcome them with the help of the people prepared for it.
\end{abstract}

\section{Introducción}

Es muy común escuchar que la educación comienza por casa, lo cual es, sin duda alguna, una verdad. Si bien es cierto, las Instituciones Educativas son las encargadas de la educación de las personas, sin embargo, es necesario entender e internalizar la idea que el aporte de ellas a la educación de los niños y adolescentes no es más que un complemento a las enseñanzas que reciben en sus hogares. La inclusión de la bioética como eje transversal en la EBR, en las escuelas se brinda los conocimientos y los valores pero es en el hogar donde se verán reforzados.

La importancia de los ejes transversales ha sido vital por el nuevo enfoque que representan; estos fueron introducidos en los sistemas educativos gracias a la publicación del "Informe de la Comisión Internacional sobre la Educación para el Siglo XXI" por la UNESCO (2006).
La transversalidad significó un cambio de paradigma en cuanto a la concepción que se tenía de la educación, pasando de un sistema educativo en el cual las enseñanzas se restringían a los contenidos de cada materia, era simplemente una transmisión de conocimientos de matemáticas, literatura, ciencias, entre otros; para pasar a un sistema educativo que, sin dejar de lado estos conocimientos, incluirían dentro del plan educativo la enseñanza de valores y actitudes que les permitirán ampliar sus conceptos e ideas sobre aspectos sociales, convivenciales y relacionados al desarrollo de la persona en su sentido más amplio². (Argueta, 2004).

En este contexto es que se señaló que, con la inclusión de la bioética como eje transversal en los planes educativos de Educación Básica Regular (EBR) se esperará obtener un mejor

\footnotetext{
${ }^{2}$ Argueta, M. (2004). Ejes Transversales en el Currículo de la Formación Inicia de Docentes. COORDINACIÓN EDUCATIVA Y CULTURAL CENTROAMERICANA. Disponible http://unpan1.un.org/intradoc/groups/public/documents/icap/unpan039733.p df
} 
futuro, no solo en la política y el aspecto social, sino también en el sentido de humanidad que ahora tendrían nuestros ciudadanos.

Una de las formas más comunes de vulnerar la dignidad humana de los estudiantes de EBR es a través del ya conocido fenómeno de Bullying, el cual con el paso de los años ha ido ganando mayor protagonismo en las escuelas, y no por el hecho de que su existencia sea reciente, sino porque cada vez son más las víctimas que se atreven a denunciarlos.

Al hablar de bullying, nos estamos refiriendo al acoso entre estudiantes, el cual es un fenómeno social cuya prevención necesita principalmente que en las instituciones educativas en las cuales exista una activa promoción del ejercicio y respeto de los derechos individuales y colectivos de los estudiantes, de igual forma se deberá promover también las responsabilidades de los mismos y la capacidad de reconocerse como sujetos capaces de actuar, participar y emitir opinión, todo ello sin que se originen ni toleren situaciones de discriminación. ${ }^{3}$ (MINEDU, 2012)

\section{La Bioética}

La Bioética desde su sentido más amplio, es una ciencia conductual porque estudia la conducta humana, no analiza los fenómenos naturales ni lo que hacen los seres vivos no conscientes. Su campo no se limita a la actividad en los campos de la investigación biomédica y la atención en salud, si no que se extiende a toda la actividad humana que se dirija la vida, porque el bien que busca tutelar es precisamente LA VIDA. Así se incorporan los atentados contra el medio ambiente, el racismo, la discriminación, el genocidio, etc. (Papacchini, 2009)

${ }^{3}$ Ministerio de Educación. (2012). Prevención e Intervención Educativa frente al Acoso entre Estudiantes. Dirección de Tutoría y Orientación Educativa. Recuperado

file:///C:/Users/Usuario/Downloads/bioetica\%20bullyn\%203.pdf
La Bioética Personalista se caracteriza por tener a la persona como centro, surge del universo, en la sociedad y en la historia, esto debido a que es precisamente la persona humana quien resume y da significado al ser del mundo, el hombre se constituye como el centro de la sociedad. (Sgreccia, 1994)

Asimismo, la persona humana es fundamento y criterio ético. De ello se pueden distinguir dos sentidos, un subjetivo y un objetivo. La persona es criterio ético en sentido subjetivo, porque una acción se convierte en ética solo en el momento que esa acción expresa una elección de la persona. $Y$ en sentido objetivo porque la persona es fundamento, medida y término de la acción moral. Dicho de otra manera, una acción es ética si respeta a plenitud la dignidad de la persona humana como los valores que están intrínsecamente inscritos en su naturaleza. (Burgos, 2013)

La concepción desde la perspectiva personalista de la Bioética, yendo de un personalismo ontológico genera un choque cultural que a su vez es el que genera su propio enriquecimiento. Es así que, será imposible prescindir de una antropología de referencia dentro de la cual el valor de la vida física corpórea, del amor conyugal y de la procreación, del dolor y de la enfermedad, de la muerte y del morir, de la relación libertad-responsabilidad, individuo y sociedad, encuentren su propio marco y su valoración ética. (Sgreccia, 1994)

Teniendo claro que la bioética se orienta a la defensa de la vida y la dignidad humana y que lucha contra cualquier acción que pueda representar una forma de lesionarla, es que se incluye también las situaciones en que viven los estudiantes de EBR que son víctimas de Bullying, tal vez la Bioética no les dará una solución inmediata para ese determinado problema que enfrentan, pero les ayudará a poder enfrentarla. 
El Bullying es un tipo de violencia que generalmente está oculto frente a los ojos de las personas en edad adulta, y que sin embargo, es bastante común y familiar entre entre los alumnos; es precisamente por ese motivo que resulta más complejo el proceso de identificarlo y aún más poder eliminarlo. Las formas en las que puede manifestarse son muchas y no se restringe a lo físico, pues también puede ser de forma verbal, con actos discriminatorios y de exclusión, sean estos de forma directa o indirecta ${ }^{4}$. (Gabaldón, 2005)

El hecho de incluir la Bioética en la EBR, ayudará a evitar el Bullying en la medida que, los estudiantes teniendo el conocimiento previo de lo que engloba la Bioética, al hacer un juicio crítico de la situación en que se encuentren, sea como víctimas o como espectadores, podrán darse cuenta que los actos que realizan los alumnos agresores vulneran su dignidad $y$ atentan contra su misma vida; consecuentemente, al ser conscientes de dicha situación, podrán pedir la ayuda necesaria y acudir con las personas adecuadas, lo cual representará una de las formas más idóneas para la erradicación de esta problemática.

\section{Problemática de los estudiantes de EBR}

Los estudiantes de EBR enfrentan una infinidad de retos y complicaciones, los cuales son abordados muchas veces desde un aspecto psicológico, pero que, a decir verdad, muy pocas veces logran solucionarse de esa manera. Los hogares disfuncionales, familias monoparentales, ausencia de una imagen de autoridad en el hogar, problemas socioeconómicos, situaciones de violencia familiar, son algunos de los contextos más comunes en que viven los estudiantes y que repercuten en su desempeño

\footnotetext{
${ }^{4}$ Gabaldón S. (2005). Sobre la violencia en las aulas: El fenómeno "Bullying". Institut Borja de Bioética. Recuperado de: file:///C:/Users/Usuario/Downloads/Bioetica\%20y\%20bullyn\%2001.pdf
}

escolar, pero sobre todo en su desarrollo personal. (Curi, 2018)

El aprendizaje de la Bioética, poniendo a la persona humana como centro y fin último de todo lo que acontece, permitirá que los estudiantes enfrenten de una mejor manera y con mayor orientación la problemática que pudieran estar viviendo.

De igual forma, la enseñanza de la Bioética en la EBR, permite también disminuir aquellos casos de violencia escolar, más conocido como Bullying. De acuerdo a lo señalado por el Ministerio de Educación, en el año 2018, se registraron 9500 casos de violencia escolar en todo el Perú, cifra que aumentó en un $70 \%$ respecto del año 2017. ${ }^{6}$ Estos datos demuestran un claro incremento, y a pasos agigantados, de esta problemática escolar y social, la cual se ha convertido en una situación más común y frecuente en los colegios; sin embargo muchas de las víctimas de este tipo de violencia no se atreven a denunciar las agresiones por miedo o por estar bajo la amenaza de sus agresores.

Es necesario que tengamos clara la definición del Bullying, este debe entenderse como el acoso escolar (también conocido como hostigamiento escolar, matonaje escolar $o_{\text {, }}$ incluso, por su término inglés bullying) es cualquier forma de maltrato psicológico, verbal o físico producido entre escolares de forma reiterada a lo largo de un tiempo determinado. Estadísticamente, el tipo de violencia dominante es el emocional y se da mayoritariamente en el aula y patio de los centros escolares ${ }^{7}$.

Esta situación que describimos, entre algunas otras que signifiquen una alteración en

\footnotetext{
${ }^{5}$ Curi, M. (2018). Las familias disfuncionales y su incidencia en el feminicidio en Lima Sur 2017-2018. Recuperado de: https://alicia.concytec.gob.pe/vufind/Record/AUTO_4ac51 ce4cca008f8e470df 173 bbed9d6

6 Ministerio de Educación. (2019). Casos de bullying escolar fueron reportados en 2018 en todo el Perú. Recuperado de: https://peru21.pe/peru/minedu-9-500-casos-bullying-escolar-reportados-perunndc-463683-noticia/? ref $=$ p21r

Fernández, C. (2015). El Bullying. Recuperado de: http://www.eduinnova.es/dic09/bullyng.pdf
} 
el correcto e ideal desarrollo de los estudiantes de EBR se pueden contrarrestar y ser superadas con la enseñanza de la Bioética Personalista a través de sus principios.

Los Principios de la Bioética Personalista y su papel en la problemática que enfrentan los estudiantes de EBR

Teniendo claro la concepción respecto a la Bioética Personalista y habiendo quedado clara la justificación de la persona como centro de la misma, veremos los cuatro principios que adquieren sentido desde esa perspectiva y cómo si estos se incluyen como ejes transversales en la EBR pueden ayudar a los estudiantes a superar la situación problemática del bullying; estos principios son: el valor fundamental de la vida, el principio de totalidad o principio terapeútico, el principio de libertad y de responsabilidad, y el principio de socialización y subsidiariedad.

\section{Principio valor fundamental de la vida}

Respecto a este primer principio, explica Sgreccia que, la vida corpórea y física del hombre no es nada extrínseco o que esté fuera de la persona, sino que representa el valor fundamental de la persona, sin importar la forma cómo se defina. Es valor fundamental porque, aunque la persona no se restringe únicamente a su cuerpo, éste valor es esencial a la misma debido a que se constituye en el fundamento único por el cual la persona se realiza y entra en el tiempo y en el espacio. A través de él expresa otros valores como la libertad, la sociabilidad y el mismo proyecto de futuro. (Sgreccia, 1999)

Gracias a este principio es que los estudiantes podrán diferenciar cuándo una acción de quienes lo rodean está dañando su integridad física, también podrá comprender que determinada situación por la que está atravesando representa una lesión sobre su corporeidad, su vida y a la vez sobre su dignidad. Solo si los estudiantes son capaces de identificar el daño que están sufriendo en la misma escuela podrán ponerle un alto, pedir ayuda y tomar las medidas necesarias para restablecer el estado original anterior a la conducta lesiva de la que fue víctima.

\section{Principio de Libertad y Responsabilidad}

Este segundo principio necesita una breve aclaración; se debe tener presente que el derecho a la vida es preexistente al derecho a la libertad, lo cual se justifica en que, para tener libertad, ser libres, necesariamente se debe tener vida, estar vivo; en conclusión, la vida es condición indispensable del ejercicio de la libertad. Por este principio se sanciona el deber moral de que la misma persona contribuya en la salvaguarda de su propia vida y la de los demás.

Nadie puede obligar a nadie a hacer algo que no quiere, como tampoco puede obligarse a nadie a asumir responsabilidades que no son consecuencias de sus propias decisiones.

El empapar la EBR de estos principios, permitirán que los estudiantes puedan establecer un juicio crítico de cada situación que perciban o a la que estén expuestos y evalúen si la conducta del otro, que es una manifestación de su voluntad, no trasgrede la voluntad de ellos ${ }^{8}$. (Sgreccia, 1999)

Los estudiantes al internalizar esas ideas, serán conscientes que nadie puede obligarlos hacer algo que no quieren o que vaya en contra de su voluntad. Por otro lado, también serán conscientes que son libres, que tienen derecho a autodeterminarse, que pueden decidir por ellos

\footnotetext{
${ }^{8}$ Sgreccia, E., Manuale di Bioetica. Fondamenti ed etica biomedica, Vita e Pensiero, Milano, 19942, pág. 52
} 
mismos, pero que eso lleva consigo el asumir las consecuencias que podrían generarse sin importar si sean buenas o malas; es decir que tendrán claro la idea de que son libres para actuar y a su vez son responsables de las consecuencias de sus actos. ${ }^{9}$

Frente a situaciones en las que, en la escuela, algún compañero pretenda obligarlos a realizar algo con lo que ellos no están de acuerdo o que signifique lesionar la autonomía de su voluntad, podrán reaccionar a tiempo para evitar que se produzcan consecuencias irreversibles, y recibirán el máximo apoyo por parte de los educadores. Serán plenamente conscientes que nada ni nadie podrá atentar contra su vida o su dignidad humana. (Sgreccia, 1999)

\section{Principio de Totalidad o Principio Terapéutico}

Este es uno de los principios más clásicos que la bioética ha adquirido por medio de la ética médica. Encuentra su fundamento en la realidad de que la corporeidad humana es un todo unitario que es resultado de la conjunción de distintas partes que se unifican de forma jerárquica y orgánica en la existencia única y personal. De esta forma al aplicar el principio terapéutico no se contradice, sino que se refuerza el principio de salvaguarda de la vida humana. (Sgreccia, 1999)

Las Instituciones Educativas enseña que nuestro cuerpo es solo nuestro, y nosotros decidimos sobre él, desde la bioética y con este principio lo que se pretende es precisamente reafirmar la protección de la vida humana.

Claro está que, el bullying y muchas de las manifestaciones de disfuncionalidad familiar, como lo es la violencia familiar, atentan y dañan el aspecto psicosomático; frente a esto, los

\footnotetext{
9 Mazo, H. (2011). La Autonomía: Principio Ético Contemporáneo. Recuperado de: file:///C:/Users/LENOVO/Downloads/Dialnet-LaAutonomia$5123760 \% 20(1) . p d f$
}

estudiantes de EBR estarán en las condiciones de reconocer cualquier acto que intente siquiera vulnerar su integridad física, y en estos casos, se hace aún más necesaria la intervención de los educadores para resguardar su bienestar en totalidad.

\section{Principio de Socialización y Subsidiariedad}

La premisa de este principio promueve que, toda persona singular encuentra su propia realización en su participación en la realización de sus semejantes. Se considera la propia vida y la de quienes nos rodean como un bien que además de ser personal también es social y se exige a la comunidad a promover el bien común promoviendo el bien de cada uno. Bajo este principio surge una obligación social de garantizar el bienestar y la salud de los ciudadanos aún a costa de restar bienes a los que se encuentren en buena situación económica o estén sanos. En la misma línea de este concepto es que nace el de subsidiariedad, mismo que ganó protagonismo con el Tratado de Maastrich, y que es originado por la doctrina social cristiana. (Sgreccia, 1999)

Por medio del principio de la subsidiariedad tenemos que, la autoridad pública debe actuar subsidiariamente para con los grupos sociales, respetando su ámbito de autonomía y no intentando suplantar o sustituir las iniciativas libres de los distintos grupos, es decir solo actuará directamente sólo cuando advierte que los particulares o los organismos intermedios no la realizan adecuadamente.

Estos principios representan uno de los más importantes logros que se tendrían con la inclusión de la bioética en la EBR a través de la transversalidad; el principio de socialización les enseñará a los estudiantes de EBR que la esencia de la humanidad está precisamente en la búsqueda del bien común, en la ayuda mutua para garantizar el bienestar propio y de nuestros 
semejantes. Se entiende que, en base a este principio, el interés general siempre estará por encima del interés individual.

El principio de subsidiariedad alcanzará su máxima expresión en la propuesta de este artículo. Este principio señala que el Estado como autoridad pública de la sociedad, es quien tiene el papel de actuar cuando algo no está funcionando como debería hacerlo.

El problema del bullying que aqueja a los estudiantes de EBR es consecuencia de los aspectos negativos de nuestra sociedad, son el resultado de la pérdida de principios y valores dentro de los hogares. Es conocido que, a través del Ministerio de la Mujer y Poblaciones Vulnerables se han realizado muchísimos programas orientados a luchar contra esa situación, sin embargo, no ha sido suficiente para superarlos. Al no poder solucionar las personas por ellas mismas esta situación es que el Estado deberá asumir la responsabilidad.

Gracias al aprendizaje de estos principios de la bioética personalista a través de los ejes transversales en los planes educativos, los estudiantes de EBR tendrán los medios necesarios para enfrentar las diversas situaciones que vulneren su dignidad humana, tales como lo son la disfuncionalidad familiar y el bullying.

Finalmente, podemos afirmar que, la inclusión de la Bioética en la educación significaría que los estudiantes estarán en la aptitud para reconocer cuándo alguien está yendo en contra de su dignidad a través de estas conductas.

\section{Conclusiones}

Los estudiantes de EBR enfrentan no solo cambios fisiológicos propios de la etapa que atraviesan, sino que también se enfrentan a diversas situaciones que forman parte de su contexto, entre los que destaca el acoso escolar, más conocido como Bullying.

La transversalidad en los planes educativos en el Perú representa un gran avance en el sistema de educación, puesto que fue una transición a un sistema que no solo se restringe a la adquisición de contenidos curriculares, sino que ahora también abarca un ámbito orientado a la mejora social.

$>$ El implementar en los planes educativos la bioética y sus principios como eje transversal representaría una verdadera intervención del Estado para contrarrestar la problemática que enfrentan los estudiantes de EBR, ésta implementación se convierte en un claro ejemplo del principio de subsidiariedad, misma que también sería comprendida por los estudiantes y será consciente que ante algún vacío respecto al tratamiento de los diversos problemas que se pudiesen suscitar, el Estado le deberá brindar los medios idóneos para superarlos.

> A través de la inclusión de los principios de la Bioética Personalista en esta transversalidad, los estudiantes de EBR podrán afrontar aquellas situaciones que signifiquen una vulneración a su dignidad, y además ayudarán a superarlas.

\section{Bibliografía}

Argueta, M. (2004). Ejes Transversales en el Currículo de la Formación Inicial de Docentes. Coordinación Educativa y Cultural Centroamericana. Disponible en http://unpan1.un.org/intradoc/groups/publi c/documents/icap/unpan039733.pdf 
Bandrés, M. Perú, Bioética y Medicina, su Enseñanza: Apuntes de una Realidad. Disponible en http://sisbib.unmsm.edu.pe/bvrevistas/spmi /v15n3/bioetica_peru.htm

Botero, C. (2006). Los ejes transversales como instrumento pedagógico para la formación en valores. Politécnica 3era edición. Disponible en: http://132.248.9.34/hevila/Revistapolitecnic a/2006/no3/5.pdf

Campos, P. (2019). Bioética General. Maestría en Bioética y Biojurídica. Universidad Católica Santo Toribio de Mogrovejo

Curi, M. (2018). Las familias disfuncionales y su incidencia en el feminicidio en Lima Sur 2017-2018. Recuperado de: https://alicia.concytec.gob.pe/vufind/Record

De Los Ríos, F. (2003). Bioética y educación: educación para la bioética. Biblioteca Virtual universitaria y otros ensayos hermenéuticos. Medellín: Universidad Pontificia Bolivariana.

Fernández, C. (2015). El Bullying. Recuperado de: http://www.eduinnova.es/dic09/bullyng.pdf

Gabaldón S. (2005). Sobre la violencia en las aulas: El fenómeno "Bullying". Institut Borja de Bioética. Recuperado de: file:///C:/Users/Usuario/Downloads/Bioetica $\% 20 y \% 20$ bullyn\%2001.pdf

Mazo, H. (2011). La Autonomía: Principio Ético Contemporáneo. Recuperado de:
file://C:/Users/LENOVO/Downloads/DialnetLaAutonomia-5123760\%20(1).pdf

Ministerio de Educación. (2019). Casos de bullying escolar fueron reportados en 2018 en todo el Perú. Recuperado de: https://peru21.pe/peru/minedu-9-500casos-bullying-escolar-reportados-perunndc-463683-noticia/?ref $=p 21 r$

Ministerio de Educación. (2012). Prevención e Intervención Educativa frente al Acoso entre Estudiantes. Dirección de Tutoría y Orientación Educativa. Recuperado de: file:///C:/Users/Usuario/Downloads/bioetica \%20bullyn\%203.pdf

Papacchini, Á. (2009). El porvenir de la ética: la autonomía moral, un valor imprescindible para nuestro tiempo. Revista de estudios sociales, (05), 32-49

Sgreccia, E. (1994). Manuale di Bioetica. Fondamenti ed etica biomedica, Vita e Pensiero, Milano.

Sgreccia, E. (1999). Manuale di Bioetica I. Fondamenti di etica biomedica. Milano: Vita e Pensiero.

Unesco Organización de la Naciones Unidas para la Educación, la Ciencia y la Cultura. Declaración Universal sobre Bioética y Derechos Humanos. División de ética de las ciencias y de las tecnologías. Sector de ciencias humanas y sociales. 1 , rue Miollis 75732 Paris Cedex 15 - Francia. 2006 